\title{
New Decentralized Control of Interconnected Systems
}

\author{
Amal Zouhri, Ismail Boumhidi
}

\begin{abstract}
In this paper, we present a new decentralized $\mathrm{H} \infty$ control for interconnected systems, the interconnected system consists of several subsystems. The proposed approach based on Lyapunov functional and a $\mathrm{H} \infty$ criterion, employed to reduce the effect of interconnections between subsystems. In the first, we study the stability of the global system in closed loop using a criterion $H \propto$, the stability conditions are presented in terms of LMI. In the second, to improve this approach, a Finsler's lemma is used for the stability analysis by LMIs. Some sufficient conditions, ensuring all the closed-loop stability are supplied in terms of Linear Matrix Inequalities (LMIs), and the new feedback gain matrix of each local controller is obtained by solving the LMIs. Finally, the practice examples are given to illustrate the efficiency of the present method.

Keywords : Interconnected system, Hळ control, decentralized state feedback, linear matrix inequality (LMI).
\end{abstract}

\section{INTRODUCTION}

In Microelectronics and power electronics, there are several dynamic systems in this field, their systems are classified in the large dimension, also their physical configuration is more complex [1-2], for the control and the modelization for this system, we composite this system in interconnected subsystems, these decompositions facilitate the resolution of the issue of stability [? ]. The synthesis of law control and stabilization can be ensuring the global safety of the system and responding in a more secure of the system [4-5], Moreover, in the phase of the modeling, the classical approach can be very hard and complicated to use to these complex systems in the context of the reliability and practical conception, this method of decentralized control is alternative to assert the stabilization of the complex and large systems [6-7-8-9].

In this paper, we focus to develop a new approaches and to extend the conditions of stability for the class of interconnected subsystems based on the Lyapunov approach and on Finsler's lemma. A Ho criterion is employed to minimize the effects of interconnections between subsystems.

Revised Manuscript Received on April 27, 2020.

* Correspondence Author

Amal Zouhri*, Department of physics, Sidi Mohammed Ben Abdellah University, Faculty of Sciences Dhar EL Mahraz, LEESI Laboratory, B.P. 1796 Fès-Atlas, 30003, Fez, Morocco. Email: amal.zouhri@usmba.ac.ma

Ismail Boumhidi, Department of physics, Sidi Mohammed Ben Abdellah University, Faculty of Sciences Dhar EL Mahraz, LEESI Laboratory, B.P. 1796, Fès-Atlas, 30003, Fez, Morocco. Email: ismail.boumhidi@usmba.ac.ma

(C) The Authors. Published by Blue Eyes Intelligence Engineering and Sciences Publication (BEIESP). This is an open access article under the CC BY-NC-ND license (http://creativecommons.org/licenses/by-nc-nd/4.0/)
The stability conditions are presented in terms of LMIs [10-11]. In order to improve this approach, a Finsler's lemma is used for the stability analysis by LMIs, with better results than the quadratic stability of LMIs, but with extra matrices, which allows a certain relaxation in the stability analysis, by obtaining a larger feasibility region. A new set of $\mathrm{N}$ decentralized controllers will thus be synthesized to ensure the stability of the global system in a closed loop.The seat of this work is arranged as following: in section 2, an overview of system description have been provided. Main results has been introduced in section 3 . In section 4 , we present numerical example to show the usefulness of the proposed results. Finally, the paper is concluded by brief conclusion in section 5 .

\section{SYSTEM DESCRIPTION}

We consider the following interconnected systems (Fig. 1), where the $\mathrm{i}^{\text {th }}$ subsystem is given by:

$$
\dot{x}_{i}(t)=A_{i} x_{i}(t)+\sum_{j=1, j \neq i}^{N} A_{i j} x_{j}(t)+B_{i} u_{i}(t), i, j \in\{1, \ldots, N\}
$$

with $x_{i}(t) \in \mathfrak{R}^{n_{i}}$ and $x_{j}(t) \in \mathfrak{R}^{n_{j}}$ are the states of the $\mathrm{i}^{\text {th }}$ and the $\mathrm{j}^{\text {th }}$ subsystem, $u_{i}(t) \in \mathfrak{R}^{m_{i}}$ is the control input. The system matrices $A_{i}, A_{i j}$ and $B_{i}$, are known real constant of appropriate dimensions

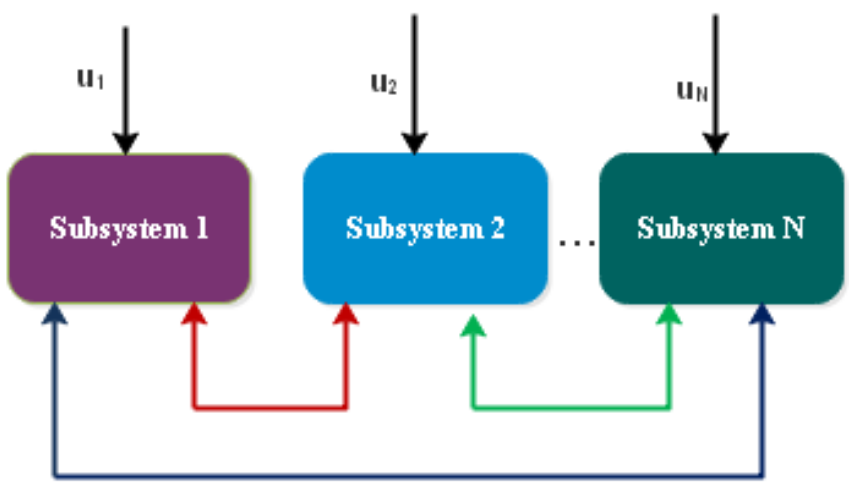

Fig. 1.Flowshart of decentralized control issue of the interconnected system .

The closed-loop subsystem is given by

$$
\dot{x}_{i}(t)=A_{c i} x_{i}(t)+\sum_{j=1, j \neq i}^{N} A_{i j} x_{j}(t), i, j \in\{1, \ldots, N\}
$$

where $u_{i}(t)=k_{i} x_{i}(t)$,

$$
A_{c i}=A_{i}+B_{i} k_{i} \text {, }
$$

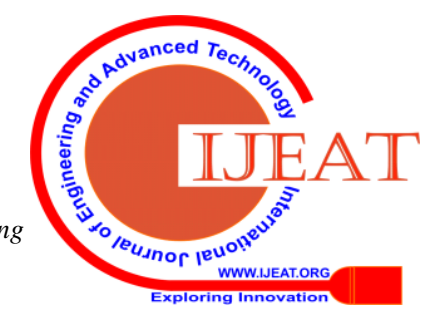


First, lemma is given which is essential for later evelopment.

Lemma 1. The Schur complement converts a class of convex nonlinear inequalities that appears regularly in control problems to an LMI. We have [12]

$$
\left[\begin{array}{ll}
Q & S \\
S^{T} & R
\end{array}\right] \succ 0
$$$$
R \succ 0, \quad Q-S R^{-1} S^{T} \succ 0 \text {, }
$$

equivalent

to

where $Q$ and $R$ are symmetric.

Lemma 2. [13] For any $n \times n$ matrix $Q$, any constant $\varepsilon \succ 0$ and any symmetric positive matrix $P \succ 0$,

$$
2 x^{T} Q y \leq \varepsilon x^{T} Q P^{-1} Q^{T} x+\varepsilon^{-1} y^{T} P y
$$

For all $x, y \in \mathfrak{R}^{n}$

Lemma 3. [14] Consider $\xi \in \mathfrak{R}^{n}, Q=Q^{T} \in \mathfrak{R}^{n \times n}$ and $\beta \in \mathfrak{R}^{m \times n}$ such that $\operatorname{rank}(\beta) \prec n$, and $\beta^{\perp}$ a basis for the null-space of $\beta$ (i.e. $\beta^{\perp} \beta=0$ ).

$$
\begin{aligned}
& \text { (1) } \xi^{T} Q \xi \prec 0 \text {, for all } \xi \neq 0, \beta \xi=0 \text {; } \\
& \text { (2) } \beta_{\perp}^{T} Q \beta_{\perp} \prec 0 \text {; } \\
& \text { (3) } \exists \chi \in \mathfrak{R}^{n \times m}: Q+\chi \beta+\beta^{T} \chi^{T} \prec 0
\end{aligned}
$$

\section{MAIN RESULTS}

In this part, the principal objective is to create a new decentralized controller guarantee the stability of interconnected systems represented by (2).

\section{A. Decentralized state feedback $H \infty$ control}

In this subsection, our objective is to provide a method of synthesis of decentralized control laws allow both the stability asymptotic of each of the interconnected subsystems and reduce the effect of interconnections between subsystems. To realize this, we are based on criterion $\mathrm{H} \infty$ to minimize the impact of $\mathrm{j}$ subsystem $(i=1,2,3, \ldots, N$, and $i \neq j)$ on the jth subsystem. these criteria are given as for $j=1,2,3 \ldots, N$.

$\int_{0}^{\infty} x_{i}^{T}(t) x_{i}(t) d t \prec \gamma_{i}^{2} \int_{0}^{\infty} \phi_{i}^{T}(t) \phi_{i}(t) d t$

where

$\phi_{i}(t)=\sum_{j=1, j \neq i}^{N} A_{i j} x_{j}(t)$

with $\gamma_{i} \succ 0$ is the performance of Hळ level.

Moreover, the vector $\phi_{i}(t)=\sum_{j=1, j \neq i}^{N} A_{i j} x_{j}(t)$ give the impact of $a \mathrm{j}^{\text {th }}$ subsystem on $\mathrm{i}^{\text {th }}$, where $\gamma_{i}$ is performance if $\mathrm{H} \infty$ is minimize.

This method makes the stabilizing the system in formula (2) with the decentralized controller $u_{i}(t)=k_{i} x_{i}(t)$, and minimize the impact of interconnections between different subsystems.

Theorem 1. The issue of decentralized Hळ control for the $\mathrm{j}^{\text {th }}$ subsystem number (2) is resolvable if $\exists$ the resolvable positive matrix $P_{i}=P_{i}^{T} \succ 0, y_{i}$ and scalars $\gamma_{i}$ positive satisfying the condition LMI :

Where $\gamma_{i}$ is minimize.

$$
\begin{aligned}
& {\left[\begin{array}{ccc}
\frac{1}{N-1}\left(P_{i} A_{i}^{T}+A_{i} P_{i}+B_{i} y_{i}+y_{i}^{T} B_{i}^{T}\right) & A_{i j} & P_{i} \\
A_{i j}^{T} & -\gamma_{i}^{2}(2 N-3) A_{i j}^{T} A_{i j} & 0 \\
P_{i} & 0 & \frac{-1}{N-1}
\end{array}\right] \prec 0} \\
& \text { Where, the local matrix is } \\
& \qquad k_{i}=y_{i} P_{i}^{-1}
\end{aligned}
$$

\section{Proof}

The stability of the system (2) for closed-loop under the criterion $\mathrm{H} \infty$ satisfy:

$\sum_{i=1}^{N}\left[\dot{V}_{i}(t)+x_{i}^{T}(t) x_{i}(t)-\gamma_{i}^{2} \phi_{i}^{T}(t) \phi_{i}(t)\right] \prec 0$

The $\mathrm{H} \infty$ (3) can be reform as:

$$
\int_{0}^{\infty} x_{i}^{T} x_{i} d t \prec \gamma_{i}^{2} \int_{0}^{\infty}\left[\sum_{j=1, j \neq i}^{N}(N-1)\left(x_{j}^{T} A_{i j}^{T} A_{i j} x_{j}\right)+\sum_{l=1, l \neq i, l \neq j}^{N} x_{l}^{T} A_{i l}^{T} A_{i l} x_{l}\right] d t
$$

Then, the system (2) is stable if:

$$
\sum_{i=1}^{N}\left[x_{i}^{T}(t)\left(A_{c i}^{T} P_{i}^{-1}+P_{i}^{-1} A_{c i}\right) x_{i}(t)+x_{i}^{T}(t) x_{i}(t)-\gamma_{i}^{2} \sum_{j=1, j \neq i}^{N}\left((N-1) x_{j}^{T} A_{i j}^{T} A_{i j} x_{j}+\sum_{\substack{l=1, l \neq i, l \neq j}}^{N} x_{l}^{T} A_{i l}^{T} A_{i l} x_{l}\right]\right) \prec 0
$$


Now, the principal objective is to reformulate inequality (9) in terms of LMI.

$$
\sum_{j=1, j \neq i}^{N}\left(\vartheta_{i j}+\sum_{l=1, l \neq i, l \neq j}^{N} \vartheta_{i l}\right)=(N-1) \sum_{j=1, j \neq i}^{N} \vartheta_{i j}
$$

the inequality (9) becomes:

$$
\begin{array}{r}
\sum_{i=1}^{N}\left[x_{i}^{T}(t)\left(A_{c i}^{T} P_{i}^{-1}+P_{i}^{-1} A_{c i}\right) x_{i}(t)+\sum_{j=1, j \neq i}^{N} x_{j}^{T}(t) A_{i j}^{T} P_{i}^{-1} x_{i}(t)+x_{i}^{T}(t) P_{i}^{-1} \sum_{j=1, j \neq i}^{N} A_{i j} x_{j}(t)+x_{i}^{T}(t) x_{i}(t)\right. \\
\left.-\gamma_{i}^{2}(2 N-3) \sum_{j=1, j \neq i}^{N} x_{j}^{T}(t) A_{i j}^{T} A_{i j} x_{j}(t)\right] \prec 0
\end{array}
$$

The equation (10) can be reform as:

$$
\sum_{i=1}^{N} \sum_{i=1, j \neq i}^{N}\left[\begin{array}{c}
x_{i}(t) \\
x_{j}(t)
\end{array}\right]^{T}\left[\begin{array}{cc}
\pi_{i} & P_{i}^{-1} A_{i j} \\
A_{i j}^{T} P_{i}^{-1} & v_{i}
\end{array}\right]\left[\begin{array}{l}
x_{i}(t) \\
x_{j}(t)
\end{array}\right] \prec 0
$$

Where,

$$
\begin{gathered}
\pi_{i}=\frac{1}{N-1}\left(A_{c i}^{T} P_{i}^{-1}+P_{i}^{-1} A_{c i}+I\right) \\
v_{i}=-\gamma_{i}^{2}(2 N-3) A_{i j}^{T} A_{i j}
\end{gathered}
$$

The formula (11) can be verified $\forall x_{i}$ and $\forall x_{j}$ if, for $i, j=1,2,3 \ldots, N$, with $j \neq i$

$$
\left[\begin{array}{cc}
\frac{1}{N-1}\left(A_{c i}^{T} P_{i}^{-1}+P_{i}^{-1} A_{c i}+I\right) & P_{i}^{-1} A_{i j} \\
A_{i j}^{T} P_{i}^{-1} & -\gamma_{i}^{2}(2 N-3) A_{i j}^{T} A_{i j}
\end{array}\right] \prec 0
$$

Multiplying (12) by $\left[\begin{array}{cc}P_{i}^{T} & 0 \\ 0 & I\end{array}\right]$ in the left and $\left[\begin{array}{rr}P_{i} & 0 \\ 0 & I\end{array}\right]$ in the right and using the Schur formula, the equation (12) satisfy

$$
\left[\begin{array}{ccc}
\frac{1}{N-1}\left(A_{c i} P_{i}+P_{i} A_{c i}^{T}\right) & A_{i j} & P_{i} \\
A_{i j}^{T} & -\gamma_{i}^{2}(2 N-3) A_{i j}^{T} A_{i j} & 0 \\
P_{i} & 0 & \frac{-1}{N-1}
\end{array}\right] \prec 0
$$

Using the change of variables

$$
y_{i}=k_{i} P_{i}
$$

inequality (13) is equivalent to the LMI (5). This completes the proof.

\section{B. New decentralized $\mathrm{H}_{\infty}$ control}

In this section, a new decentralized $\mathrm{H} \infty$ control conditions for the interconnected closed-loop system (2) is developed. The main result for interconnected system is stated in the following theorem.

Theorem 2. The resolution of the system (2) for decentralized $\mathrm{H} \infty \quad$ if $\quad P_{i}=P_{i}^{T} \succ 0, y_{i}, F_{i} \quad$ and $\gamma_{i}, a_{i}, b_{i}$ are positives and satisfy

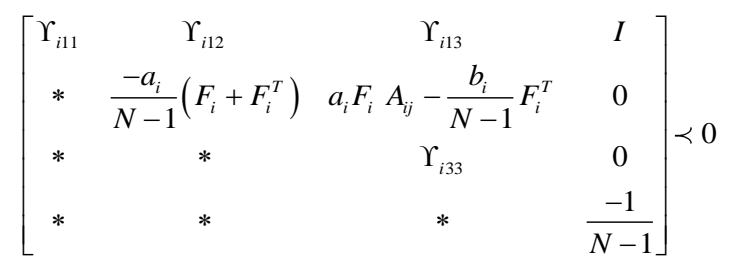

where

$$
\begin{aligned}
& \Upsilon_{i 11}=\frac{1}{N-1}\left(F_{i} A_{i}^{T}+A_{i} F_{i}^{T}+B_{i} y_{i}+y_{i}^{T} B_{i}^{T}\right) \\
& \Upsilon_{i 12}=\frac{1}{N-1}\left(P_{i}^{-1}-F_{i}-a_{i} A_{i} F_{i}^{T}+a_{i} B_{i} y_{i}\right) \\
& \Upsilon_{i 13}=F_{i} A_{i j}+\frac{b_{i}}{N-1}\left(A_{i} F_{i}^{T}+B_{i} y_{i}\right) \\
& \Upsilon_{i 33}=-\gamma_{i}^{2}(2 N-3) A_{i j}^{T} A_{i j}+b_{i} F_{i} A_{i j}+b_{i} A_{i j}^{T} F_{i}^{T}
\end{aligned}
$$

The new local matrix is

$$
k_{i}=y_{i}\left(F_{i}^{T}\right)^{-1}
$$

\section{Proof}

The stability of system (2) on closed loop satisfy the criterion $\mathrm{H} \infty$ 


$$
\sum_{i=1}^{N}\left[\dot{V}_{i}(t)+x_{i}^{T}(t) x_{i}(t)-\gamma_{i}^{2} \phi_{i}^{T}(t) \phi_{i}(t)\right] \prec 0
$$

The above inequality can be refurmuled as

$$
\begin{aligned}
& \sum_{i=1}^{N} \sum_{j=1, j \neq i}^{N}[A+B-C] \prec 0 \\
& A=\frac{1}{N-1}\left(\dot{x}_{i}^{T}(t) P_{i}^{-1} x_{i}(t)+x_{i}^{T}(t) P_{i}^{-1} \dot{x}_{i}(t)\right. \\
& \left.B=x_{i}^{T}(t) x_{i}(t)\right) \\
& C=\gamma_{i}^{2}(2 N-3) x_{j}^{T}(t) A_{i j}^{T} A_{i j} x_{j}(t)
\end{aligned}
$$

we obtained

$$
\sum_{i=1}^{N} \sum_{j=1, j \neq i}^{N} \zeta_{i}^{T} Q_{i} \zeta_{i} \prec 0
$$

where

$$
\begin{aligned}
\zeta_{i} & =\left[\begin{array}{lcc}
x_{i}^{T}(t) & \dot{x}_{i}^{T}(t) & x_{j}^{T}(t)
\end{array}\right]^{T}, \\
Q_{i} & =\left[\begin{array}{ccc}
\frac{1}{N-1} & \frac{1}{N-1} P_{i}^{-1} & 0 \\
* & 0 & 0 \\
* & * & -\gamma_{i}^{2}(2 N-3) A_{i j}^{T} A_{i j}
\end{array}\right],
\end{aligned}
$$

Expression (2) can be written as:

$$
\sum_{j=1, j \neq i}^{N}\left[\begin{array}{ccc}
\frac{1}{N-1} A_{c i} & \frac{-1}{N-1} I & A_{i j}
\end{array}\right]\left[\begin{array}{c}
x_{i}(t) \\
\dot{x}_{i}(t) \\
x_{j}(t)
\end{array}\right]=0
$$

Considering

$$
\begin{gathered}
\chi_{i}=\left[\begin{array}{c}
F_{i} \\
a_{i} F_{i} \\
b_{i} F_{i}
\end{array}\right], \\
\beta_{i}=\left[\begin{array}{lll}
\frac{1}{N-1} A_{c i} & \frac{-1}{N-1} I & A_{i j}
\end{array}\right],
\end{gathered}
$$

with

$$
\beta_{i \perp}=\left[\begin{array}{cc}
I & 0 \\
A_{c i} & (N-1) A_{i j} \\
0 & I
\end{array}\right]
$$

and using condition (ii) of lemma 3, we obtained the equality between $\beta_{i \perp}^{T} Q_{i} \beta_{i \perp}$ and the LMIs in (5).

Now, Applying Finsler's Lemma 3 in condition (3), with (19), (21) and (22) writes

$$
\begin{aligned}
& {\left[\begin{array}{ccc}
\frac{1}{N-1} & \frac{1}{N-1} P_{i}^{-1} & 0 \\
* & 0 & 0 \\
* & * & -\gamma_{i}^{2}(2 N-3) A_{i j}^{T} A_{i j}
\end{array}\right]} \\
& +\left[\begin{array}{c}
F_{i} \\
a_{i} F_{i} \\
b_{i} F_{i}
\end{array}\right]\left[\begin{array}{ccc}
\frac{1}{N-1} A_{c i} & \frac{-1}{N-1} I & A_{i j}
\end{array}\right]+(*) \prec 0
\end{aligned}
$$

Thus, (24) is equivalent to (15). This completes the proof of the lemma 2.

\section{EXPERIMENTAL RESULT}

To prove the efficacy of the proposed method, we give some numerical example extract of the literature

Application 1. We give the decentralized $\mathrm{H} \infty$ control issue of interconnected system of equation (2) with

$$
\begin{gathered}
A_{1}=\left[\begin{array}{cc}
-7 & 0 \\
-6 & 0.1
\end{array}\right], A_{12}=\left[\begin{array}{cc}
0.1 & 0 \\
0 & 0.1
\end{array}\right], A_{13}=\left[\begin{array}{cc}
0.01 & 0 \\
0 & 0.1
\end{array}\right] \\
A_{2}=\left[\begin{array}{cc}
5 & 0 \\
1 & -1
\end{array}\right], A_{21}=\left[\begin{array}{cc}
0.1 & 0 \\
0 & 0.1
\end{array}\right], A_{23}=\left[\begin{array}{cc}
0.01 & 0 \\
0 & 0.1
\end{array}\right] \\
A_{3}=\left[\begin{array}{cc}
-1 & 0 \\
0.1 & -1
\end{array}\right], A_{31}=\left[\begin{array}{cc}
0.01 & 0 \\
0.1 & 0.1
\end{array}\right], A_{32}=\left[\begin{array}{cc}
0.01 & 0.1 \\
0 & 0.1
\end{array}\right] \\
B_{1}=\left[\begin{array}{c}
0.47 \\
1.2
\end{array}\right], B_{2}=\left[\begin{array}{c}
0.47 \\
0.8
\end{array}\right], B_{3}=\left[\begin{array}{l}
0.6 \\
0.4
\end{array}\right]
\end{gathered}
$$

Using the first system theorem below system and minimize $\gamma_{i}$, we observe that for this primary subsystem

$$
\begin{aligned}
& k_{1}=\left[\begin{array}{ll}
5.5149 & -6.1398
\end{array}\right], P_{1}=\left[\begin{array}{ll}
1.7981 & 0.0260 \\
0.0260 & 1.2381
\end{array}\right], \\
& \gamma_{1}=0.2667
\end{aligned}
$$

for the secondary subsystem

$k_{2}=\left[\begin{array}{ll}-95.3551 & 22.7011\end{array}\right]$,

$P_{2}=\left[\begin{array}{ll}0.2035 & 0.5290 \\ 0.5290 & 1.7732\end{array}\right]$,

$\gamma_{2}=0.4888$

for the third subsystem

$$
\begin{aligned}
k_{3}= & {\left[\begin{array}{ll}
-7.4300 & -5.6618
\end{array}\right], P_{3}=\left[\begin{array}{ll}
1.1218 & 0.3183 \\
0.3183 & 0.7969
\end{array}\right], } \\
& \gamma_{3}=1.1014
\end{aligned}
$$

Using the second system theorem below system and minimize $\gamma_{i}$, we observe that

For the first subsystem

$k_{1}=\left[\begin{array}{ll}10.0746 & -1.8061\end{array}\right]$,

for the second subsystem

$k_{2}=\left[\begin{array}{ll}-32.5773 & 6.7252\end{array}\right]$,

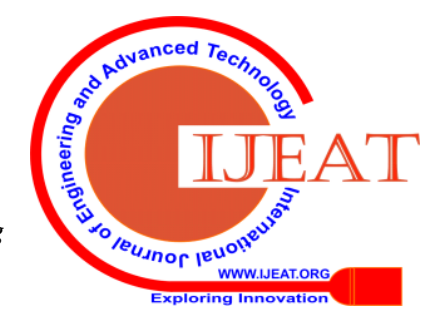


for the third subsystem

$$
k_{3}=\left[\begin{array}{ll}
-0.2800 & -0.3621
\end{array}\right]
$$

In this numerical result, we observe that the following initial conditions use these vectors
The evolution of the control system and these dynamics in a closed-loop for every each subsystem are shown in Figures 1 to 4.

$$
x_{1}(0)=\left[\begin{array}{c}
1 \\
-0.5
\end{array}\right], \quad x_{2}(0)=\left[\begin{array}{l}
1 \\
0
\end{array}\right], \quad \text { and } x_{3}(0)=\left[\begin{array}{l}
1 \\
1
\end{array}\right] \text {, }
$$

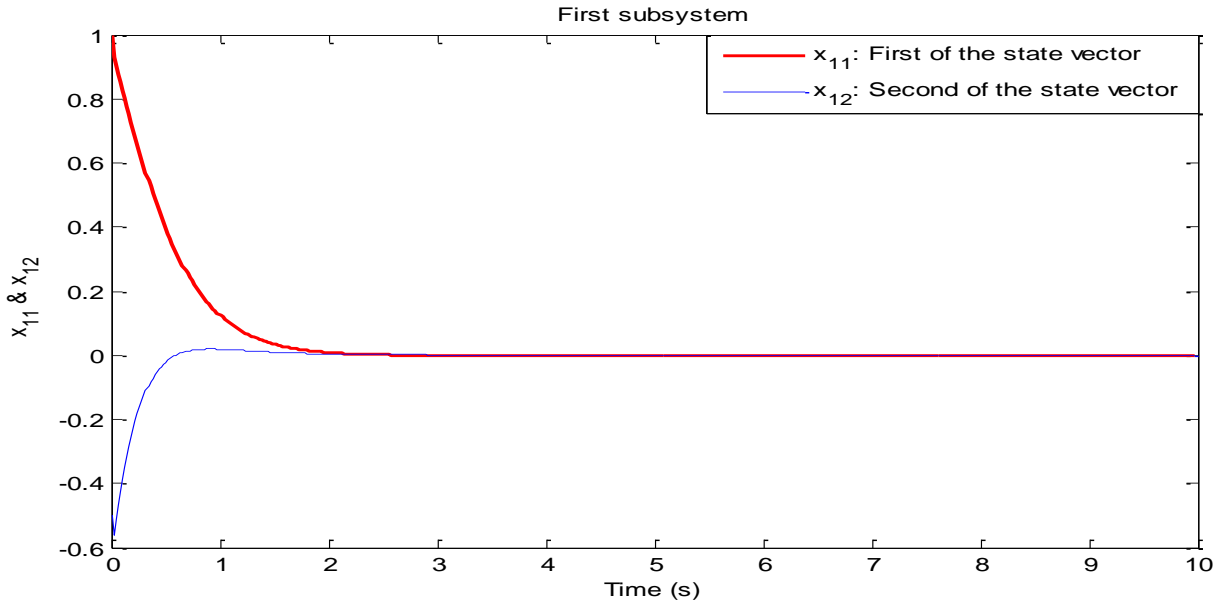

Fig. 2.a-First subsystem .

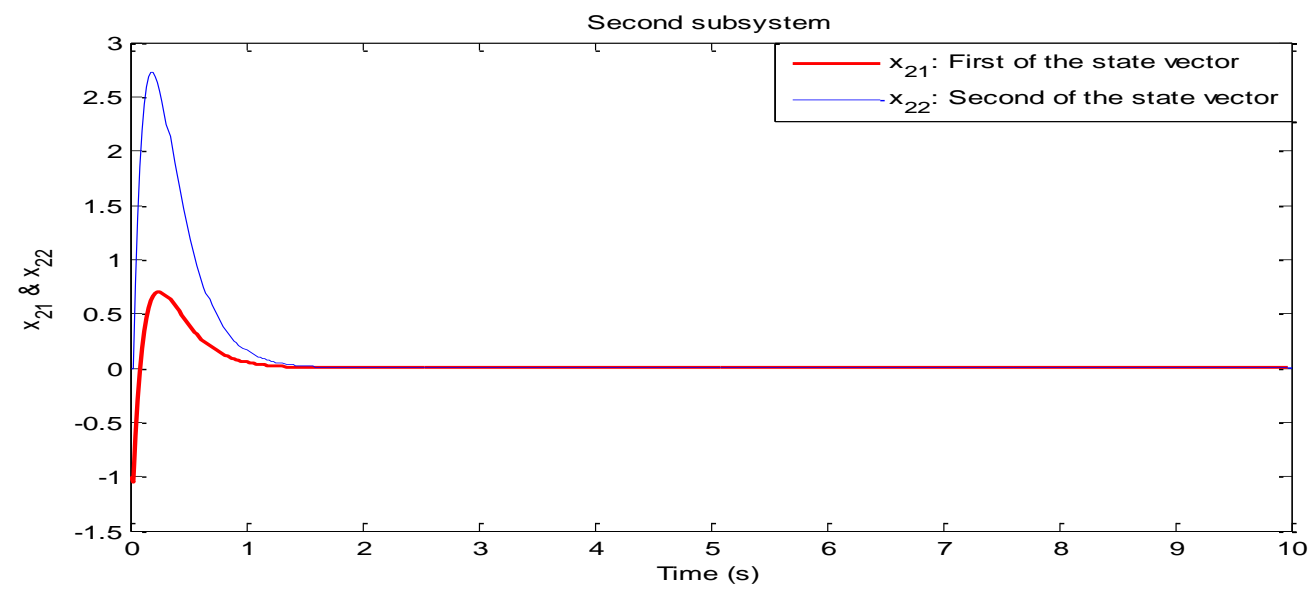

Fig. 2.b- Second subsystem.

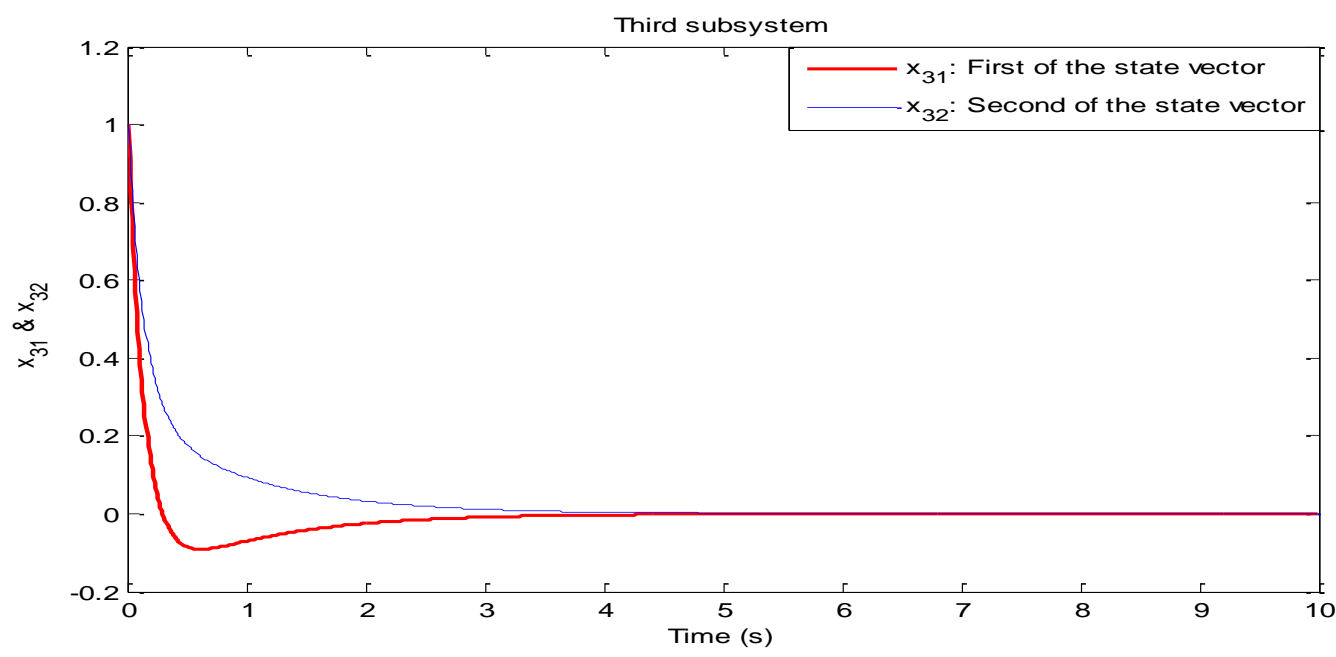

Fig. 2.c- Third subsystem.

Figures 2. Temporal evolution of the state vector of interconnected subsystem with $\mathbf{H} \infty$ control.

Published By:

Blue Eyes Intelligence Engineering

\& Sciences Publication

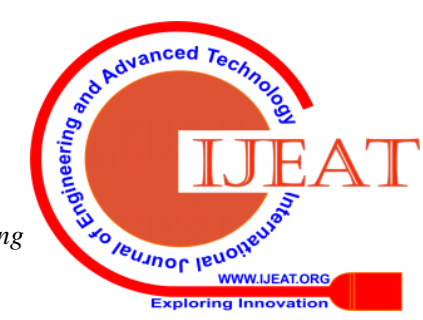


New Decentralized Control of Interconnected Systems

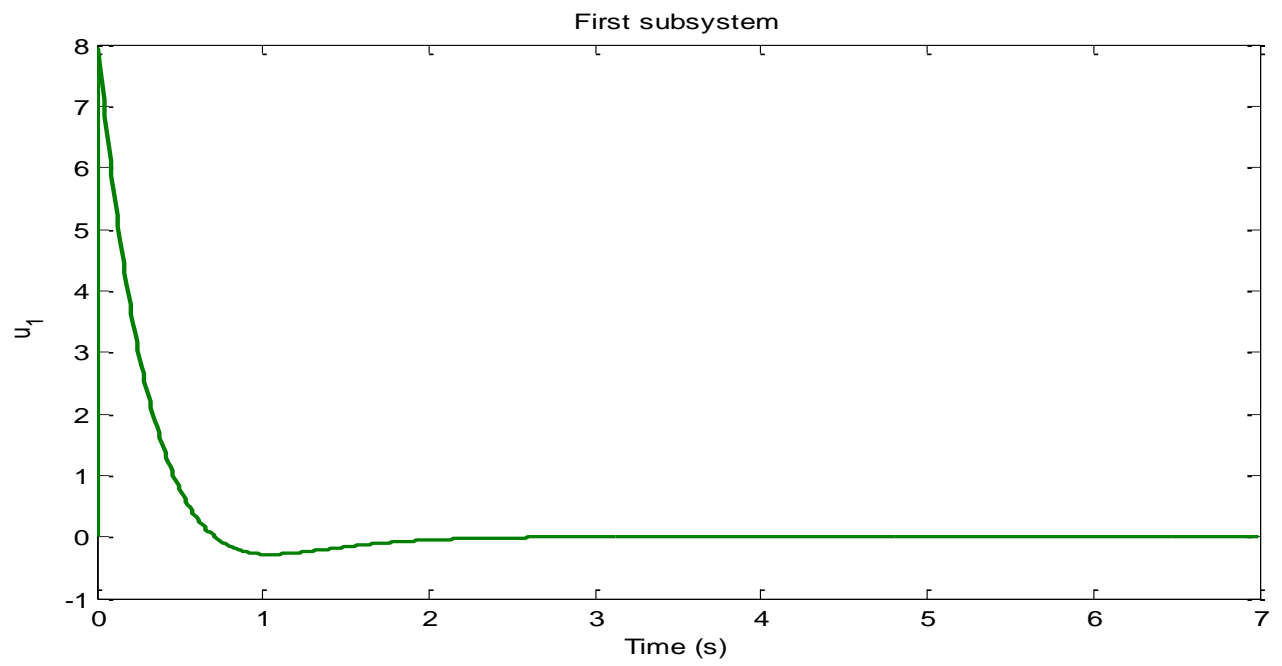

Fig. 3.a- First subsystem.

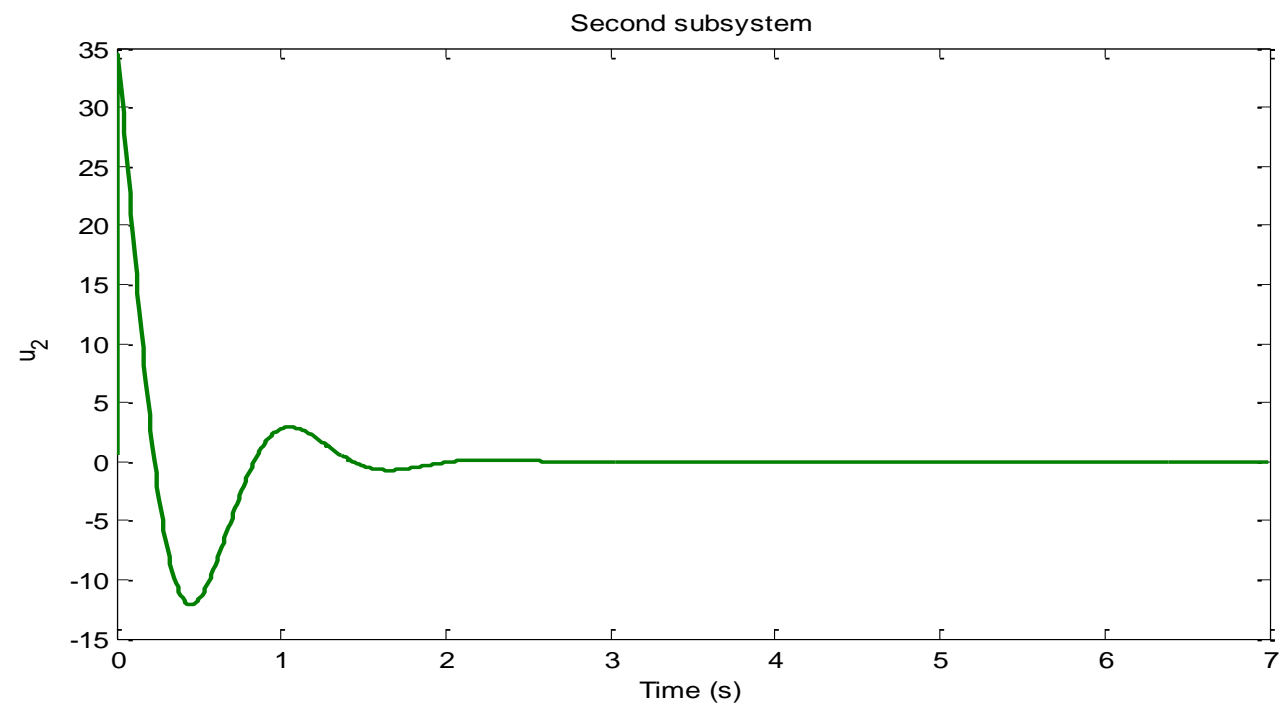

Fig. 3.b. Second subsystem

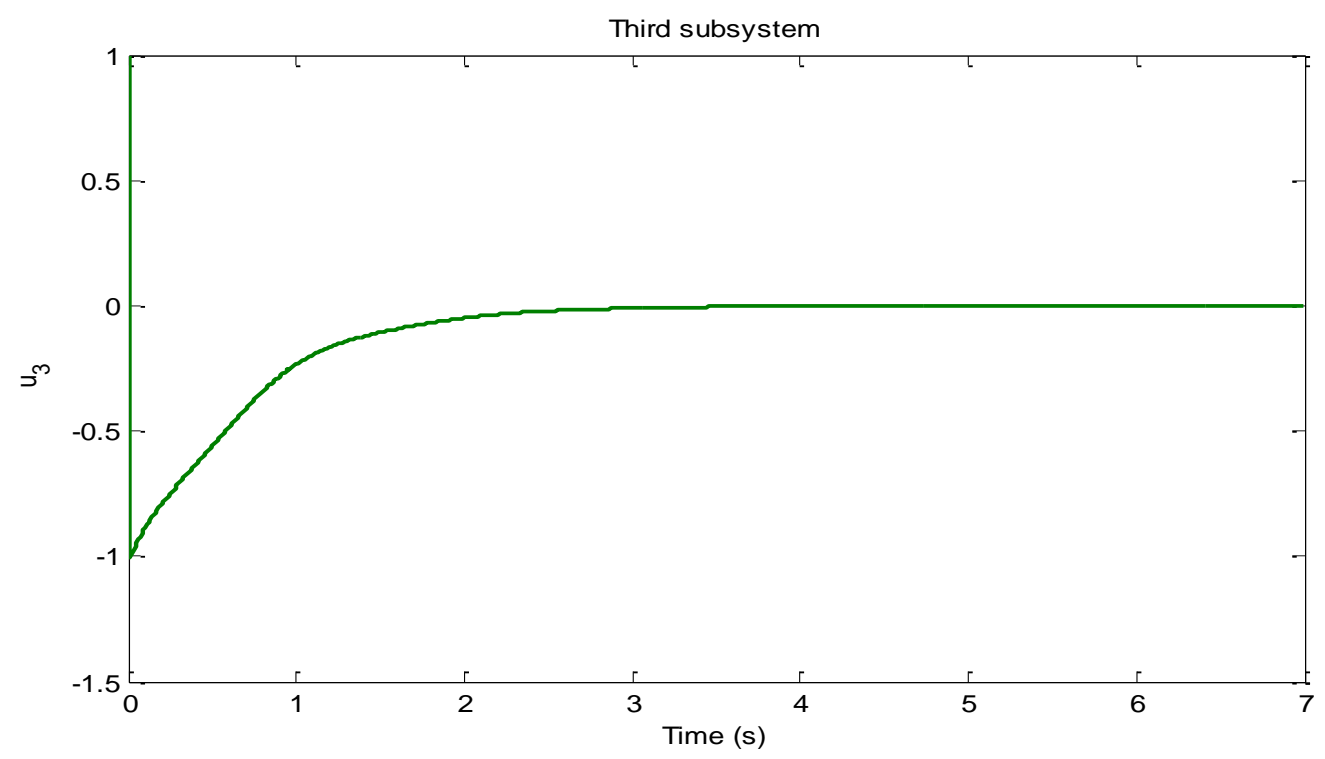

Fig. 3.c. Third subsystem

Figures 3. Temporal evolution of the control laws $(\mathrm{H} \infty \mathrm{control})$

The resulting controller stabilizes the interconnected system with an average time of convergence of $5 \mathrm{~s}$ order. 


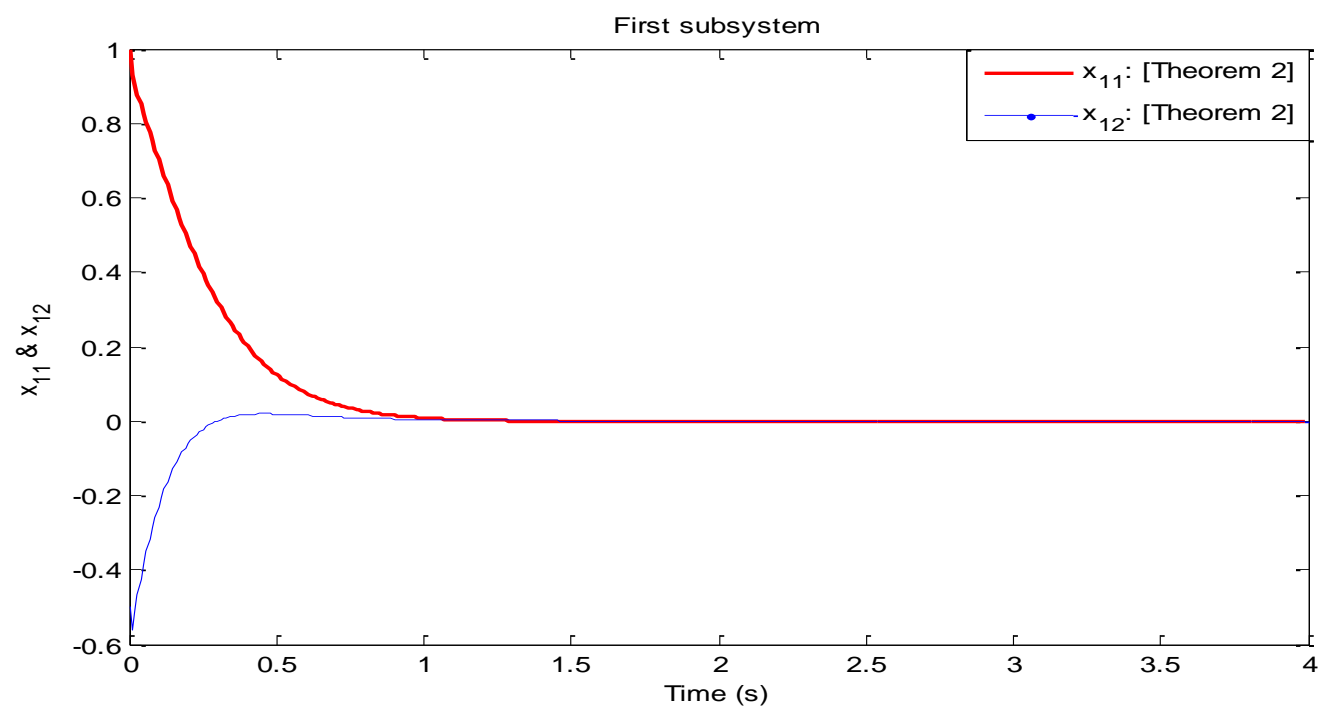

Fig. 4.a. First subsystem

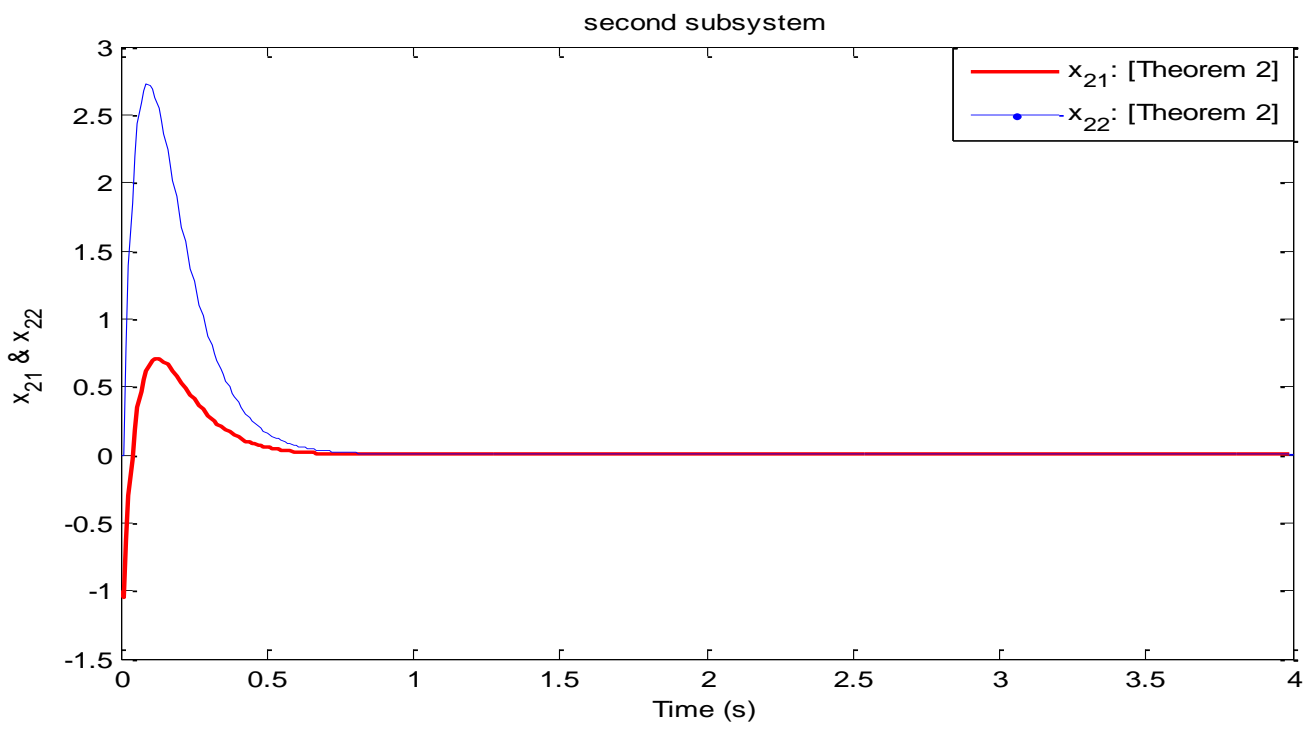

Fig. 4.b. Second subsystem

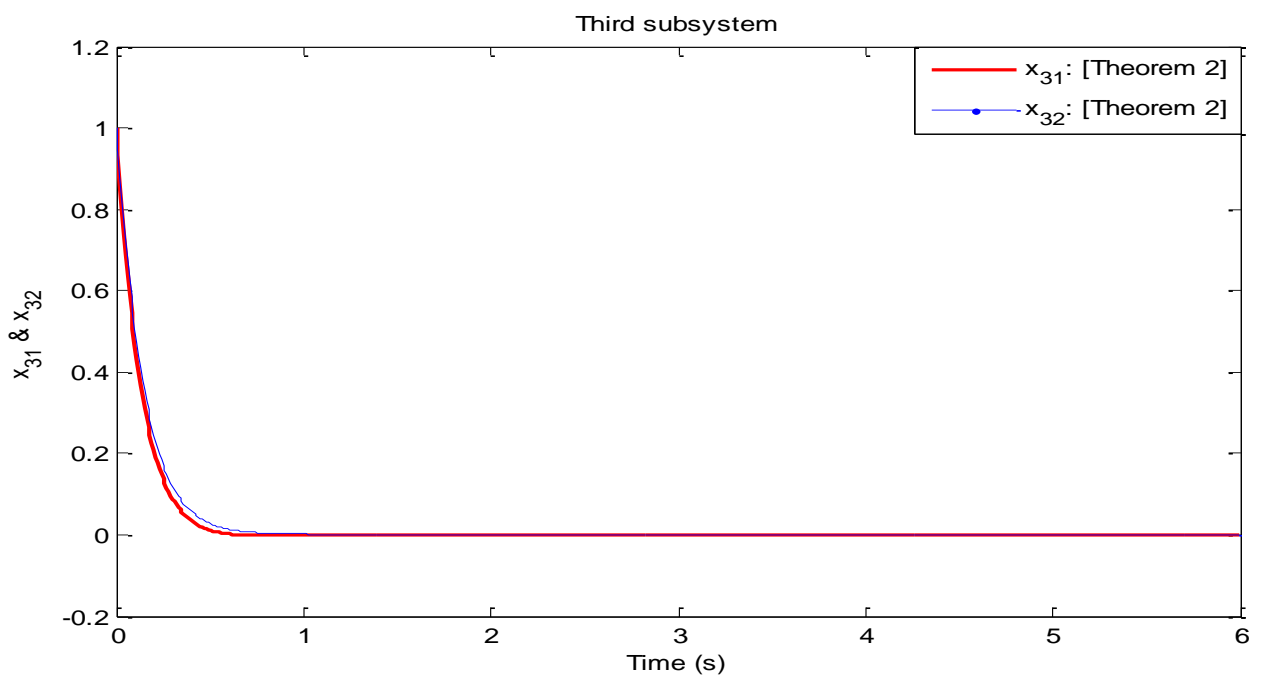

Fig. 4.c. Third subsystem

Figures 4. Temporal evolution of the state vector of interconnected subsystem with proposed method.

Published By:

Blue Eyes Intelligence Engineering

\& Sciences Publication

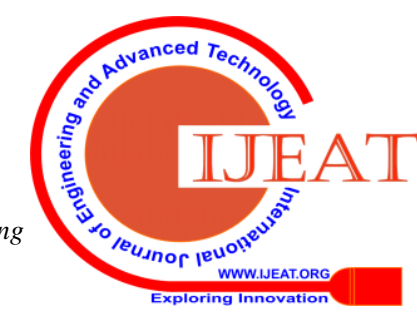


New Decentralized Control of Interconnected Systems

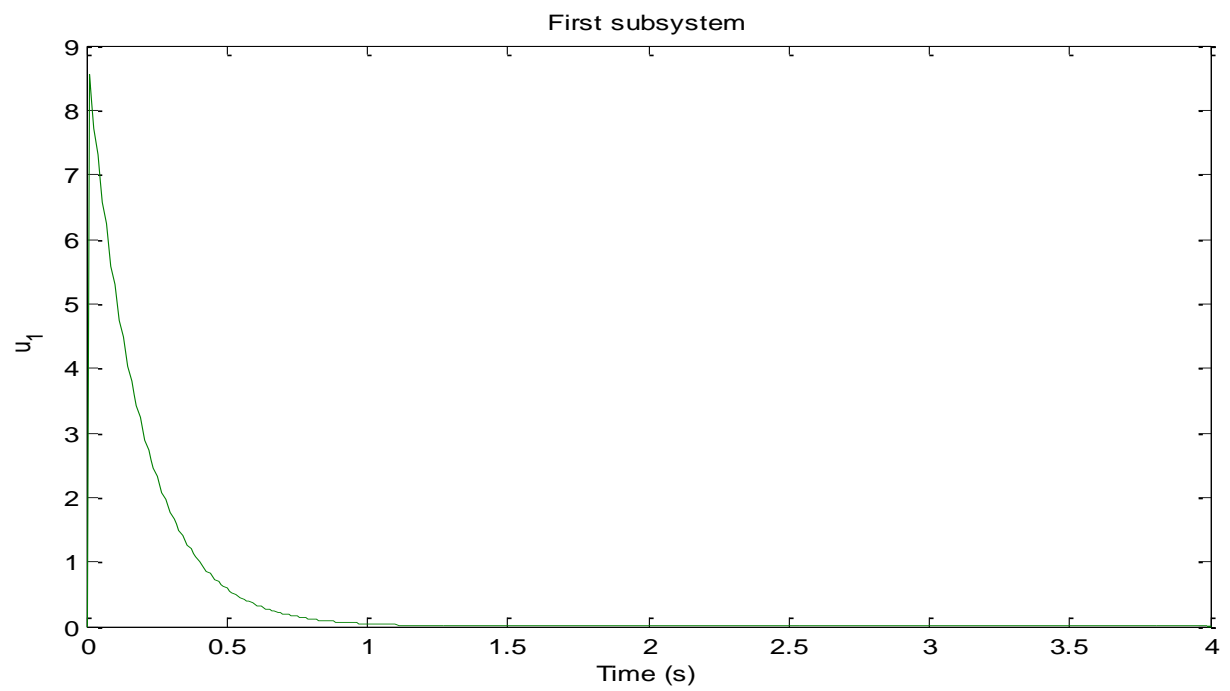

Fig. 5.a. First subsystem

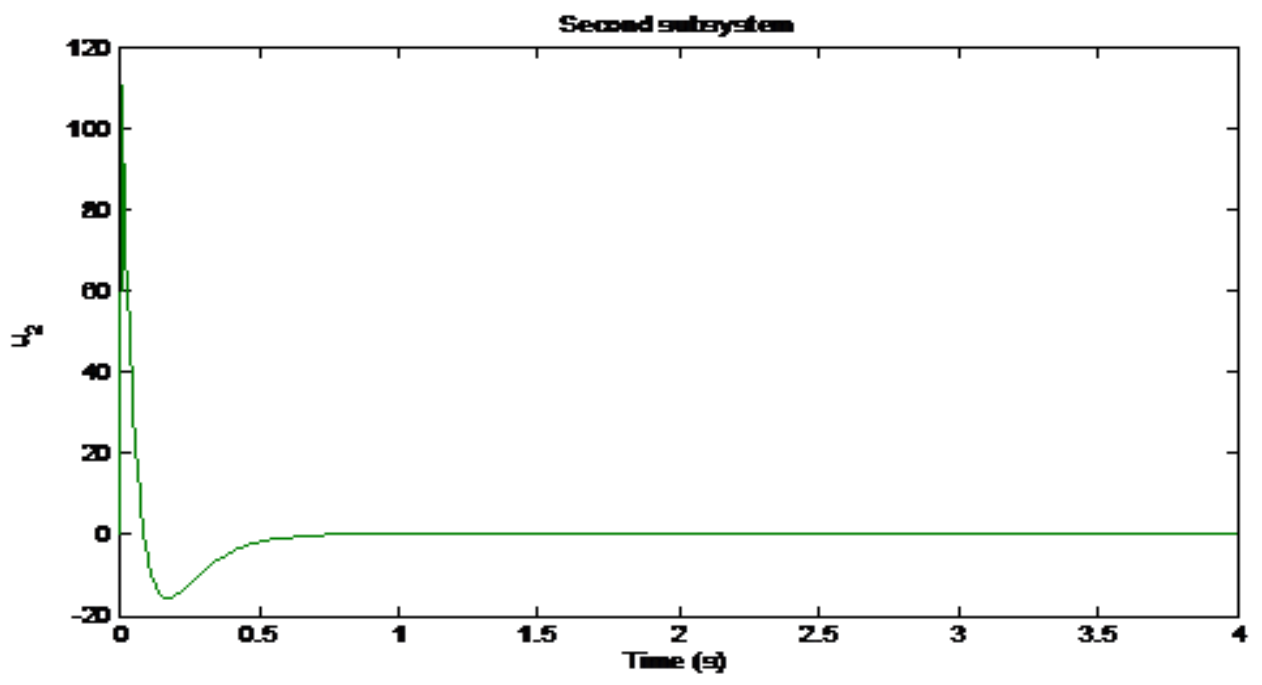

Fig. 5.b. Second subsystem

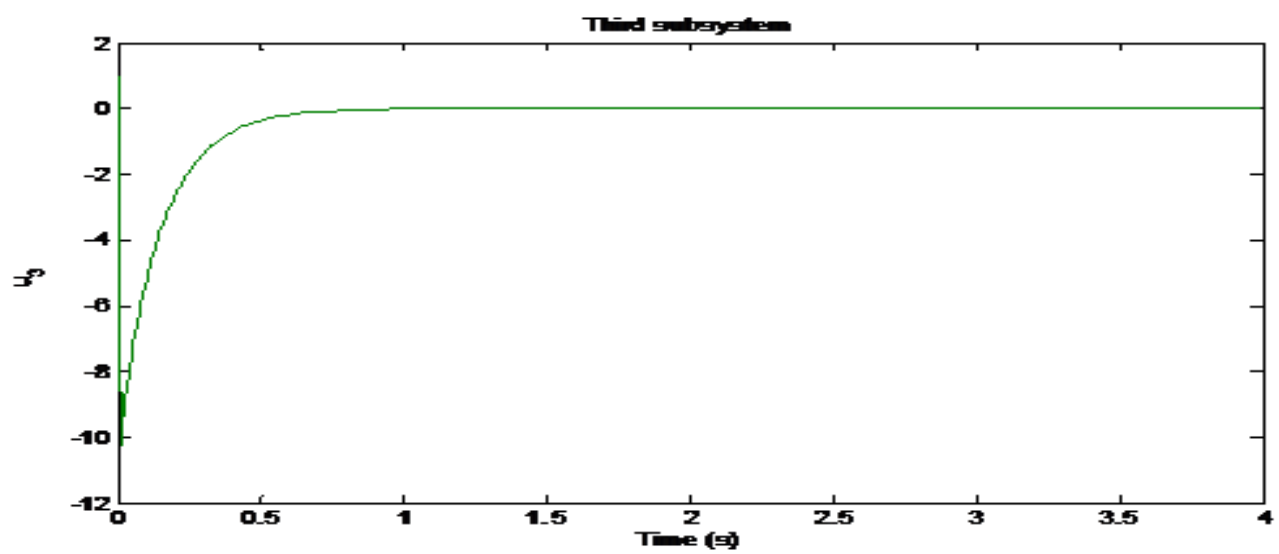

Fig. 5.c. Second subsystem

Figure 5. Temporal evolution of the control laws (proposed method)

We find that the resulting controller stabilizes the family of subsystems with an average time of convergence of the order of 1 s. We note here that each subsystem has its own convergence time. Moreover, we can also note that the dynamics of the second subsystem is faster than the first and third. These results show the effectiveness of the proposed approach in the measure where the family of subsystems are correctly stabilized by the whole of the three laws of new

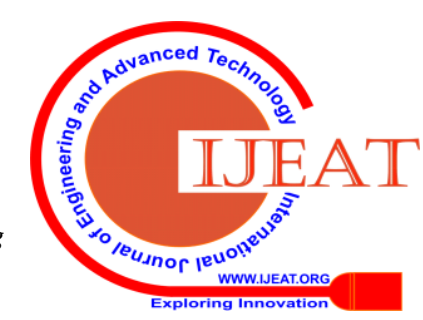


In this work, we proposed a new decentralized H $\infty$ control for interconnected systems such as the closed-loop feedback subsystems are asymptotically stable. We used a Lyapunov function candidate and a $\mathrm{H} \infty$ criterion to minimize the impact of interconnections between subsystems, applying Finsler's lemma, the sufficient conditions ensuring stability closed loop were formulated in the terms of LMI. Finally, the obtained result are given to prove the efficiency the proposed method, and the graphics result shows that the system is well stabilized.

\section{REFERENCES}

1. Mahmoud, M.S., Almutairi, N.B., (2011), Resilient decentralized stabilization of interconnected time- delay systems with polytopic uncertainties, Int. J. Robust. Nonlinear Control, Volume 21, pp. 355-372.

2. Siljak , D.D., (1991), Decentralized Control of Complex Systems. Academic Press.

3. A. ZOUHRI, I. BOUMHIDI, Decentralized Robust Hळ Control of Large Scale Systems with Polytopic-Type Uncertainty, International Review of Automatic Control, vol. 9(2), pp. 103-109, March 2016.

4. Schuler, S., Münz, U., Allgöwer, F., (2014), Decentralized state feedback control for interconnected systems with application to power systems, Journal of Process Control, Volume 24, pp. 379-388.

5. Widyan, M., (2014), Operational Performance of Synchronous Generator

Hybrid-Excited by PMDC and PV Generators, International Review of Electrical Engineering (IREE), Volume 9(4), pp. 863-872.

6. ZOUHRI, A. and BOUMHIDI, I., Decentralized control of interconnected systems with time-delays, 12th ACS/IEEE International Conference on Computer Systems and Applications AICCSA 2015 November 17-20, (2015), Marrakech, Morocco.

7. Lee, T. N., and Radovic, U. L., 1988, Decentralized Stabilization of Linear Continuous and Discrete- Time Systems with Delays in Interconnections, IEEE Transactions on Automatic Control, Volume 33, pp. 757-761.

8. Mahmoud, M.S., Almutairi, N.B., (2009), Decentralized Stabilization of Interconnected Systems with Time-varying Delays, European Journal of Control, Volume 6, pp. 624-633.

9. Xu, B., 1995, On Delay-Independent Stability of Large-Scale Systems with Time Delays, IEEE Transactions on Automatic Control, Volume 40, pp. 930-933.

10. A. ZOUHRI, M. BENYAKHLEF, S. KRIRIM, I. BOUMHIDI, Robust Stability and $\mathrm{H} \infty$ Analysis for Interconnected Uncertain Systems, International Journal of Mathematics and Statistics ${ }^{\mathrm{TM}}$, vol. 17 (1), pp.55-66, 2016.

11. A. ZOUHRI, I. BOUMHIDI, Robust Decentralized Stabilization for Uncertain Interconnected Systems, International Journal of Mathematics and Computation, vol. 27 (4), pp.48-56, 2016.

12. Olivier, B., 2008, Notions de base pour une initiation à la synthese robuste de correcteurs par approche LMI, 3.

13. Zhang, Y. and Pheng, A. H., (2002), Stability of fuzzy control systems with bounded uncertain delays, IEEE Trans. Fuzzy Syst., Volume 10, pp. 92-97.

14. Oliveira, M. C. de, Skelton, R. E., (2001), Stability tests for constrained linear systems. in: S.O. Reza Moheimani (Ed.), Perspectives in Robust Control of Lecture Notes in Control and Information Science, Springer-Verlag, New York, volume 268, pp. 241-257.

\section{AUTHORS PROFILE}

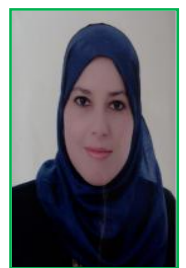

Amal Zouhri received the B. Sc., M. Sc. and Ph. D. degrees in Electrical Engineering from Faculty of Sciences, Sidi Mohammed Ben Abdellah University, Morocco in 2008, 2011 and 2017, respectively. Her research interests include Embedded System, Stability and Stabilization of Interconnected systems, Decentralized systems,

E-mail: amal.zouhri@usmba.ac.ma

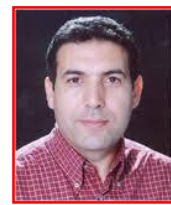

Ismail Boumhidi is a Professor and Head of physics department at the Faculty of Sciences, Fez Morocco. His research areas include adaptive robust control, multivariable nonlinear systems, and fuzzy logic control with applications. .

E-mail: ismail.boumhidi@usmba.ac.ma

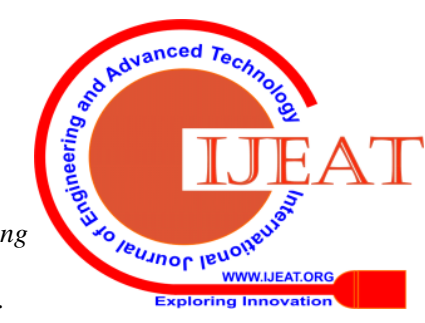

